\title{
Diseño estratégico prospectivo y enfoque económico social del turismo en zonas vírgenes \\ DOI: $10.22403 /$ UQROOMX/TYP03/09
}

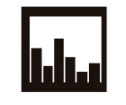

RESUMEN

Inocencio Raúl Sánchez Machado*

Víctor Manuel Figueroa Albelo**

El objetivo principal de esta investigación fue la formulación de un modelo generador de una estrategia prospectiva para el desarrollo turístico de la cayería noreste deVilla Clara. Se trató de un procedimiento inexistente en la literatura y menos aún en la práctica, que incluye el perfeccionamiento del proceso constructivo, el diseño de un modelo estratégico hotelero que responde a la categoría cualitativa de producto robusto y la sistematización de un enfoque económico social bajo condiciones de riesgo en todo el desarrollo de la región que nos ocupa. La estrategia recoge los enfoques económicos y sociales para la construcción de obras dirigidas a elevar la eficiencia general del proceso.

Palabras |Desarrollo turístico, inversión, planeación estratégica, prospectiva, CLAVE turismo de cayería.

*Universidad Central de Las Villas / raulsm@fce.uclv.edu.cu

**Universidad Central de Las Villas 


\section{Introducción}

La cayería noreste de Villa Clara comprende un conjunto de cayos e islotes ubicados al norte de esa provincia. Destacan por sus características naturales los cayos Fragoso, Las Brujas, Francés, Ensenachos y Santa María, que se encuentran en estado virginal; se distinguen por sus playas de arena blanca, aguas claras y transparentes con tonos verdes y azules en caprichosos contrastes.

La cayería se compone también de otros atractivos turísticos naturales que amplían su oferta más allá del recurso playa y las características particulares de los cayos, entre los cuales podemos citar:

- El comedero y anidamiento de flamencos

- Numerosas zonas para la pesca de cordel

- Interesantes áreas para el buceo contemplativo

- El histórico barco San Pascual construido con hormigón armado en 1920 y varado desde 1933

- Los típicos centros de acopio de langosta y peces

El presente trabajo se enmarca en el proceso de toma de decisiones con enfoque administrativo y económico. Decidir sobre el desarrollo de una nueva región requiere ordenar de forma prospectiva y holística, lo económico y social, ya que para Cuba conlleva más importancia que para otros países, considerando el riesgo.

\section{Metodología}

Se formuló un modelo generador de un diseño estratégico que contiene cinco grandes momentos, tal como lo ilustra la figura I.

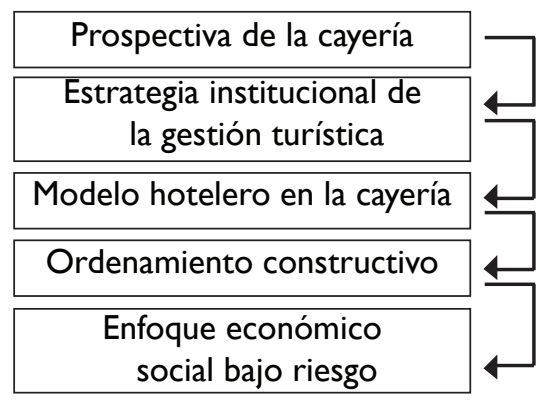

Figura I. EsQUema del MOdelo propuesto 


\section{Lineamientos estratégicos con enfoque prospectivo para el desarrollo turístico de la cayería noreste de Villa Clara}

Para aprovechar el potencial de desarrollo que ofrece la cayería noreste deVilla Clara y transformarla en un destino turístico de gran calidad, armonizado con las características naturales de la región y con los valores culturales, históricos y autóctonos tradicionales, se aplicaron los lineamientos estratégicos prospectivos para perfeccionar el Plan Director de Desarrollo Turístico de la región

A partir de septiembre de 1989 se adoptaron las siguientes decisiones estratégicas:

- Estudios de calidad sobre los grupos insulares y zonas litorales del archipiélago cubano y de líneas de base a Cayo Santa María; también con respecto a los proyectos para las poliredes, a la elaboración del primer Plan Director (1991), a la cesión de la zona a la Corporación Gaviota (1999) y al proyecto de la planta generadora (Diesel) en Cayo Fragoso.

- Calidad de las viales asociadas con el vial marítimo del Pedraplén, el vial regional al este de Santa María y el vial turístico.

- Calidad de las inversiones para la construcción de las instalaciones en Punta Periquillo (1997), del primer hotel ( 1998-200 I), del aeropuerto de Las Brujas, del segundo hotel (1999-2003), de la construcción de la planta de generación eléctrica y de la conductora de agua, del centro de comunicación y de la ampliación del aeropuerto de Santa Clara.

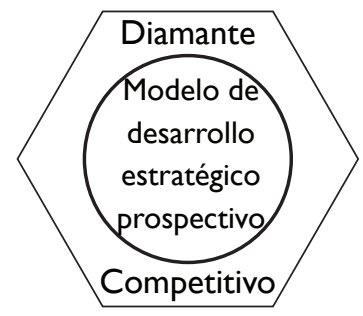

Perfeccionamiento del Plan Director de la región

FIGURA 2. DiAMANTE COMPETITIVO PROSPECTIVO 
- Calidad de los servicios del centro de comunicación, del abasto de agua y de la infraestructura, para mejor atención de los clientes.

A partir de la configuración del Diamante Competitivo de la región (véase figura 2), se logró construir un mejor perfil competitivo de la cayería noreste al identificar los factores básicos y factores avanzados, la estrategia, la estructura de la región y la rivalidad de las organizaciones, determinando con esto las condiciones imperantes en la región y las cadenas productivas para conformar la plataforma urbana y regional de la zona de nuevo desarrollo y, por último, las condiciones de la demanda para caracterizar a los compradores y a los competidores de la región.

Es determinante haber construido un perfil competitivo para la región; para lo cual se reflejan los componentes que deben formar parte de esta herramienta analítica de estudio.

Por último, se propuso el Plan de Ordenamiento Territorial de la cayería noreste, cuya primera versión fue elaborada en 1990 con la participación de todos los organismos implicados a nivel nacional y provincial.

Se incluyen en este trabajo las áreas para alojamiento turístico y capacidad habitacional, las precisiones de vialidad interna de la región, la infraestructura técnica general de soporte a la región de desarrollo, las instalaciones extrahoteleras en cada cayo y región de influencia, los accesos aéreos, terrestres y marítimos a la cayería, la conciliación medioambiental en todas sus dimensiones de desarrollo, la conciliación con los principales inversionistas de la región y, por último, la respuesta en toda su amplitud a la exigencias de las cadenas extranjeras en materia de explotación.

\section{Perfeccionamiento estratégico institucional de soporte al desarrollo turístico de la nueva región}

La presente investigación aborda el diseño de la estrategia corporativa del grupo empresarial cubano Gaviota Centro, encaminado a lograr la eficiencia y eficacia de la gestión organizacional y su mejor adaptación al entorno turístico donde está enmarcado.

Para ello, se propuso una tecnología gerencial que incluye la caracterización del objeto de estudio, el diseño de la misión, un diagnóstico estratégico 
general apoyado en el análisis situacional DAFO y la proyección de escenarios utilizando la técnica prospectiva. El punto medio fue la declaración de una visión consecuente y retadora que expresa las cualidades que ambiciona el grupo Gaviota, sustentada en los valores estratégicos, así como en el planteamiento de la estrategia general con su ventaja corporativa o estrategia de negocio y sus correspondientes estrategias operacionales. Como punto culminante de la investigación se incluye un sistema de control estratégico para garantizar el flujo informativo y la retroalimentación.

La técnica utilizada en la investigación fue el método de intervenciónacción, mediante un proceso de formación y desarrollo de equipo. Se trabajó en tiempo real, de forma iterativa, estableciendo diez sesiones de trabajo con objetivos y tiempo planificados.

Todo este estudio conduce a reafirmar la necesidad del desarrollo y crecimiento de la organización, partiendo del poder económico, el beneficio social y la diversificación de un producto y mercado que garantice la eficiencia de la gestión para el desarrollo respectivo y la exclusividad en la competencia.

\section{Perfeccionamiento del proceso preparatorio de obras constructivas en la cayería noreste de Villa Clara}

Las principales premisas que han sido definidas para perfeccionar el procedimiento de preparación de obras son la integración de los organismos bajo el principio de dirección matricial; la creación de un grupo permanente de trabajo para el mejoramiento de la proyección de obras; la realización de

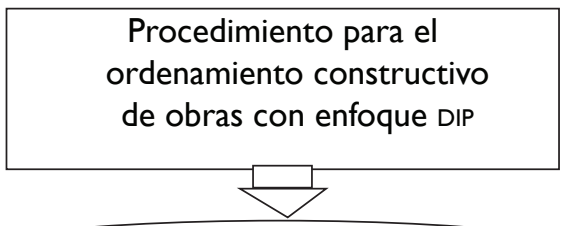

Algoritmo de análisis de perfeccionamiento constructivo en la región

Figura 3. Procedimiento para el ordenamiento constructivo CON ENFOQUe DiP 
reuniones grupales bajo el principio de brainstorming; la formación de grupos multidisciplinarios de expertos para el diseño de productos temáticos; $y$, por último, la realización de chequeos de control sistemáticos.

Se propone un algoritmo de análisis del perfeccionamiento de la preparación de obras (véase figura 3) que incluye un análisis de la prioridad de la preparación de la inversión en el polo; la evaluación de posibilidades de suprimir etapas; la introducción de la simultaneidad de las etapas del proceso; la coordinación e integración de equipos de trabajo; la confección del cronograma general de la preparación de la inversión turística; y la discusión, aprobación y contratación de los factores implicados en la preparación de las inversiones.

\section{Diseño de un modelo estratégico hotelero en calidad de Producto Robusto en la región estudiada}

Se propone y aplica un sistema estructurado con los fundamentos del modelo hotelero en calidad de Producto Robusto (véase figura 4) en relación con la inserción al medio natural; los subsistemas de alojamiento, gastronómico, recreativo, público comercial, de mantenimiento y el de las edificaciones extrahoteleras.

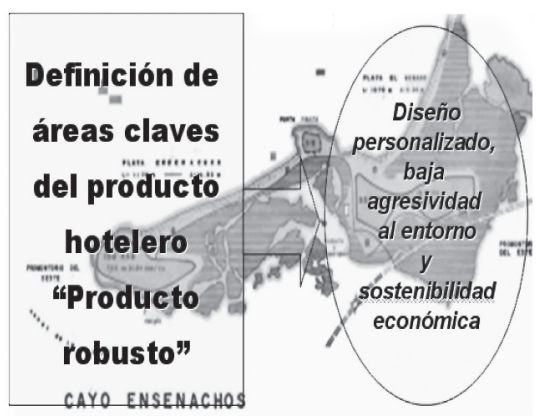

Figura 4. Fundamentos DEL PRODUCTO HOTELERO EN CALIDAD DE PRODUCTO ROBUSTO

El modelo propuesto es de amplia capacidad de réplica en regiones similares; asegura estándares de calidad exigidos por las cadenas extranjeras de explotación hotelera. 


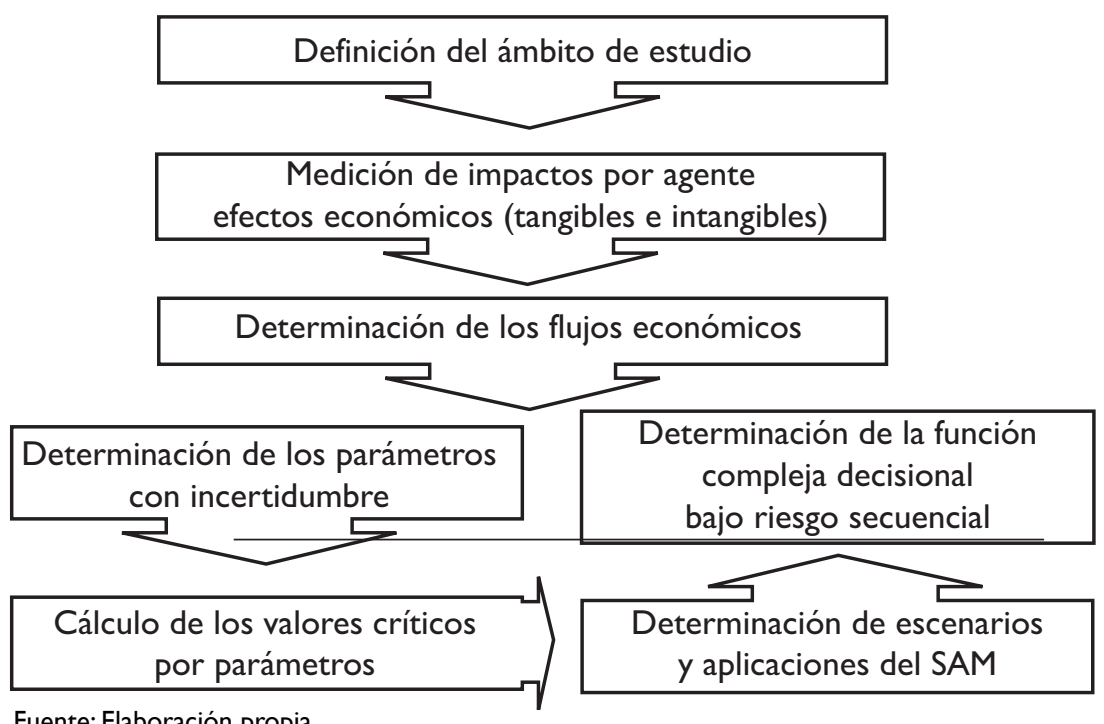

Fuente: Elaboración propia.

Figura 5. PROCEDIMIENTO PARA LA INCORPORACIÓN DEL RIESGO EN PROYECTOS HOTELEROS

\section{Enfoque económico social del desarrollo inversionista en las condiciones de la región de nuevo tipo para Cuba}

Luego de identificar la insuficiencia teórico-metodológica para un análisis de factibilidad de inversiones en una determinada región de desarrollo turístico, que permita la consideración del enfoque económico-social en la evaluación y las implicaciones del riesgo y la incertidumbre en estos análisis, se propone un esquema básico (EBEIEES) para el estudio de proyectos de infraestructura turística. Los componentes para el análisis social y bajo riesgo son con vistas a perfeccionar el proceso de evaluación del desarrollo inversionista a escala territorial.

Se tratan los fundamentos teóricos para un enfoque económico-social de evaluación de inversión en las condiciones de la economía cubana; se aborda el diseño metodológico de un esquema básico de evaluación de inversiones con enfoque económico-social bajo incertidumbre y riesgo; y por último, se expone la validación de la propuesta de esquema básico de evaluación de proyectos de inversión en el sector turístico de Villa Clara, específicamente un vial de enlace 
terrestre desde la capital villaclareña hasta el enclave turístico de la cayería noreste, un sistema de abastecimiento de agua a la misma zona y el primer hotel en esta misma región turística. Este resultado de investigación ya tiene continuidad de aplicación en un nuevo proyecto de investigación para los análisis de impacto social del desarrollo turístico en la cayería, que incorpora las dimensiones socioeconómicas, socioculturales y sociodemográficas de un análisis integrador.

\section{Conclusiones}

La cayería noreste de Villa Clara sólo ha ejecutado I $2 \%$ de las obras hoteleras que se proyectan. En su región norte, Villa Clara posee impactos potenciales aún sin estudiar.

Las inversiones extrahoteleras y de infraestructura de la cayería están incompletas y varias se encuentran en fase de estudio. Otras inversiones con enfoque regional en el país, en polos de desarrollo, requieren similares criterios de valoración prospectiva y un enfoque económico social bajo riesgo.

Los resultados de este trabajo han permitido contribuir a lograr un umbral de rentabilidad en la región a sólo I $5 \%$ de la capacidad hotelera instalada; asimismo, han permitido reducir el presupuesto de gastos del primer hotel en más de tres millones de pesos; garantizar la reducción del plazo de ejecución de obras en un hotel en cinco meses respecto del diseño inicial, y evitar el gasto de 10 millones de pesos anuales mediante la toma de decisiones de inversión correctas.

En el orden social, este trabajo contribuyó a acelerar la ubicación del turismo como renglón decisivo en la vida económica de la provincia; a ampliar la oferta de empleos directos e indirectos a 30000 profesionales de la región central del país; redujo los accidentes viales por decisiones de proyecto consistentes y aminoró el tiempo en recorridos por mejores diseños de proyectos viales.

En el contexto ambiental, tomando en cuenta los fundamentos hoteleros de Producto Robusto, el hotel Las Brujas ha recibido reconocimientos ambientales por su construcción ecológica, lo que ha dado lugar a la adopción de medidas económicas de impacto ambiental en las inversiones extrahoteleras. Por último, las definiciones estratégicas en el Plan Director han contribuido a la protección ambiental de la cayería.

La investigación-acción se orientó a la formulación de un "modelo, en calidad de procedimiento generador, de una estrategia con carácter prospectivo de la cayería noreste de Villa Clara". Algo semejante no existe en la literatura y mucho menos en la práctica regular. 
El trabajo permite perfeccionar el Plan Director de Desarrollo Turístico de esta región, que contempla, además de los enfoques económicos y sociales de los procesos inversionistas para elevar su eficiencia general, el diseño de un modelo hotelero en calidad de Producto Robusto que asegure la originalidad y competitividad esperada de dicha zona.

\section{FUENTES CONSULTADAS}

Baca, G. (1990). Evaluación de proyectos. Análisis y administración del riesgo. $2^{\mathrm{a}}$ ed. México: McGraw-Hill.

Barrios, C. (1997). Análisis coste-beneficio de las inversiones en infraestructuras: una aplicación al tercer acceso a Cádiz. Tesis doctoral no publicada. Andalucía: Universidad de Andalucía.

Brealey, R., y Myers, S. (1993). Fundamentos de financiación empresarial. $4^{\mathrm{a}}$ ed. Madrid: McGraw-Hill.

Brown, P.y Gibson, D. (1973). “A quantified model for facility site selection application to a multiplan location problem". Revista AllE Transaction, 4, I I, 52 p.

Bull, A. ( 1994). La economía del sector turístico. Madrid: Edición Alianza.

Castro, O. (199I). Evaluación financiera, económica y social. San José: Banco Centroamericano de Integración Económica.

Castro, R., y Mokate, M. (1998). Evaluación económico social de proyectos de inversión. Santa Fe de Bogotá: Edición UniAndes.

Coloma, F. (1990). Evaluación social de proyectos de inversión. La Paz:Asociación Internacional de Fomento / Banco Mundial.

De Rus, G. (200I). Análisis costo-beneficio. Barcelona:Ariel Economía.

Gramlich, E. (1990). A guide to benefit - cost analysis. 2a ed. Michigan: University of Michigan.

Rocabert, J. (1999). La evaluación de políticas y proyectos. Criterios de valoración económicos y sociales. Barcelona: Icaria \& Antrazyt.

Sánchez, I. (2003). “Enfoque económico social bajo condiciones de riesgo en la evaluación de inversiones”. Tesis doctoral no publicada. La Habana: Universidad de La Habana, Facultad de Economía.

Plan Director de Desarrollo Turístico.... ( 199 I) Plan Director de Desarrollo Turístico de la Cayería Noreste de VillaClara. Documento inédito.Villa Clara:Oficina Provincial de Planificación Física en Villa Clara, I I5 p. 

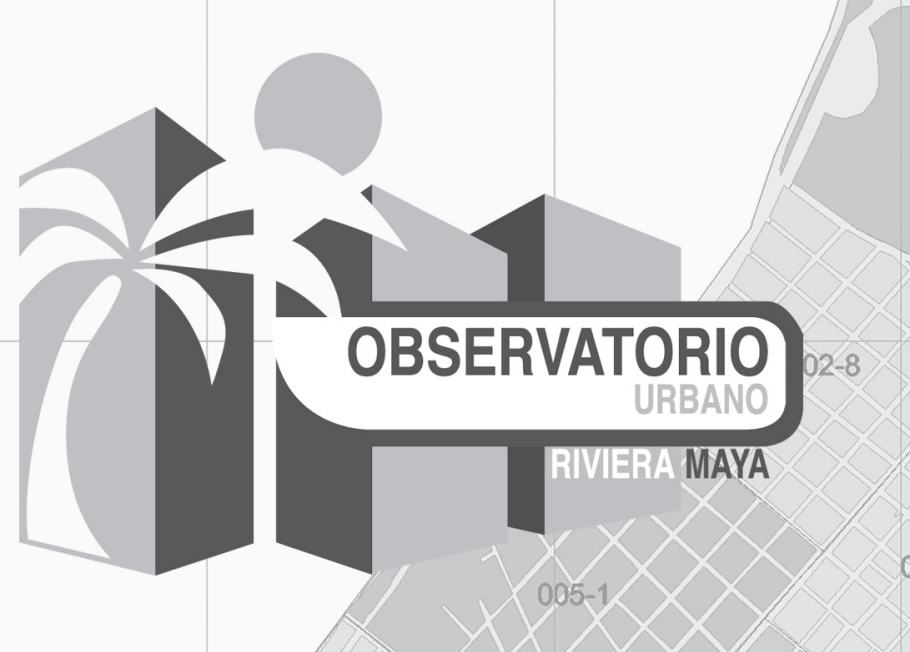

$040-3$

$082-7$

$003-2$

$083-1$

078-0

La función del Observatorio

$007-0$ Urbano de la Riviera Maya es la de generar datos e información y estimular un análisis integral,

$091-6$ pero objetivo para que la concer-

042-2 tación de actores se mueva pro-

0992 activamente hacia la superación

$044-1$ de la pobreza urbana, en estrecha $100-7$

$072-3$

$073-8$
0988 relación con los objetivos del

Desarrollo del Milenio y la implementación progresiva de $106-4$

$092-0$ la Agenda Hábitat y la Agenda 21.

\section{$090-1$}

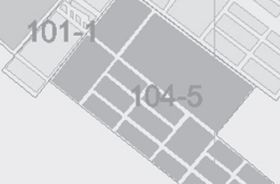

Observatorio Urbano de la Riviera Maya Universidad de Quintana Roo Unidad Académica Cozumel observatoriourbano@uqroo.mx 